\begin{tabular}{|c|c|c|}
\hline \multirow{3}{*}{$\begin{array}{r}\text { Case Reports in } \\
\text { Gastroenterology }\end{array}$} & \multicolumn{2}{|c|}{ Case Rep Gastroenterol 2013;7:44-48 } \\
\hline & $\begin{array}{l}\text { DOI: } 10.1159 / 000346299 \\
\text { Published online: January 24, } 2013\end{array}$ & $\begin{array}{l}\text { (c) } 2013 \text { S. Karger AG, Basel } \\
1662-0631 / 13 / 0071-0044 \$ 38.00 / 0 \\
\text { www.karger.com/crg }\end{array}$ \\
\hline & $\begin{array}{l}\text { This is an Open Access article license } \\
\text { NonCommercial-NoDerivs } 3.0 \text { Lice } \\
\text { online version of the article only. Di }\end{array}$ & $\begin{array}{l}\text { Is of the Creative Commons Attributio } \\
\text { er.com/OA-license), applicable to th } \\
\text { on-commercial purposes only. }\end{array}$ \\
\hline
\end{tabular}

\title{
Two Cases of Mucinous Cystadenoma of the Appendix Successfully Treated by Laparoscopy
}

\author{
Yuko Yoshida ${ }^{a} K_{\text {Koichi Sato }}{ }^{a}$ Takashi Tada $^{a}$ Hiroshi Maekawa ${ }^{a}$ \\ Mutumi Sakurada ${ }^{a}$ Hajime Orita $^{a}$ Tomoaki Ito $^{a}$ Fumiko Hirata $^{a}$ \\ Ryo Wadab \\ Departments of ${ }^{a}$ Surgery and ${ }^{b}$ Pathology, Juntendo Shizuoka Hospital, Juntendo \\ University School of Medicine, Izunokuni, Japan
}

\section{Key Words}

Mucinous cystadenoma of the appendix · Laparoscopic surgery

\begin{abstract}
Two cases of mucinous cystadenoma of the appendix successfully treated by laparoscopy are reported. An 81-year-old woman with lower right back pain was diagnosed with mucinous cystadenoma of the appendix or appendiceal carcinoma and underwent elective laparoscopic surgery. The other case involved a 70-year-old man with hematochezia who was diagnosed with mucinous cystadenoma. He also underwent elective laparoscopic surgery. In these two cases, gauze was folded around the tumors rather than grasping them directly. The postoperative courses were uneventful, and these patients had no recurrent disease at 2-year follow-up. In such cases, surgical excision of the tumor without rupture is of paramount importance because rupture of the lesion can cause pseudomyxoma peritonei. Though appendiceal mucinous cystadenoma has been considered a contraindication of laparoscopic resection, it was possible to achieve this by using a laparoscopic procedure with a gauze technique.
\end{abstract}

\section{Introduction}

Mucinous cystadenoma of the appendix is a rare condition that develops as a result of proliferation of mucin-secreting cells in an occluded appendix. It is found in $0.2-0.3 \%$ of resected appendices in Europe and the United States [1,2]. Surgical excision of the tumor without rupture is of paramount importance because rupture of the lesion causes pseudo-

\footnotetext{
Yuko Yoshida

Department of Surgery, Juntendo Shizuoka Hospital

Juntendo University School of Medicine

1129 Nagaoka, Izunokuni-shi, Shizuoka 410-2295 (Japan)

E-Mail yyoshida@juntendo.ac.jp
} 
myxoma peritonei [3, 4]. Even in benign disease such as cystadenoma, dissemination of mucin-producing cells into the peritoneal cavity can cause pseudomyxoma peritonei. Since its diagnosis in terms of malignancy or benignity is difficult before surgery, it is important to remove it without rupture of the lesion. Recently, applications of laparoscopic appendectomy have been reported [3-6]. This laparoscopic approach avoids a large incision for exploration of the peritoneal cavity and confers the advantage of minimally invasive surgery.

We describe two cases of appendiceal mucinous cystadenoma successfully treated by laparoscopic resection. It is important to plan careful resection to remove a mass. It was possible to achieve this by using a laparoscopic procedure with gauze technique. We present the procedure for handling the appendix.

\section{Case 1}

An 81-year-old woman was referred to a local hospital with lower right back pain experienced for a few days. She did not have any significant history of bloating or diarrhea, constipation or any melena or hematochezia. She denied any recent episodes of fever, nausea or vomiting and reported a stable appetite and weight. She was diagnosed with an ovarian tumor in that local hospital and then visited the Department of Gynecology in our hospital. She was then admitted to our department for further examination.

Routine laboratory examination on admission showed no abnormalities, except for tumor markers, e.g. carcinoembryonic antigen, showing a high level. Computed tomography (CT) scan revealed a $10 \times 3.5 \mathrm{~cm}$ cystic mass arising from the cecum with calcification inside (fig. 1a). Colonoscopy showed no mucosal lesion and the appendiceal orifice was normal. A barium enema study demonstrated a defect in the mesenteric side of the cecum and the appendix was not visualized.

The suspected preoperative diagnosis was mucinous cystadenoma of the appendix or appendiceal carcinoma. The patient received elective laparoscopic surgery. With the patient in the supine position, pneumoperitoneum was established with the open technique, and four trocars were placed in the upper, lower, left and right abdomen. There was no evidence of ascites and peritoneal implants. The mass was removed along with the appendix. We folded gauze around the tumor rather than grasping it directly (fig. 1b). The ileocecal vessels were exposed, clipped and divided in sequence to the root to remove the lymph nodes. By blunt dissection, a tunnel was created in the retroperitoneum in order to separate the ileal and right colon mesentery from retroperitoneal structures. The freed right colon was delivered and resected. We used a rap disk to transport the specimen through the abdominal wall (fig. 2a). Microscopic examination of the appendix showed multilocular cystadenoma. There was no evidence of dysplasia of the epithelial cells (fig. 2b). The postoperative course was uneventful and the patient had no recurrent disease at 2-year follow-up.

\section{Case 2}

A 70-year-old man was referred to a local hospital with hematochezia. There was no history of abdominal pain or change in bowel habit. He was diagnosed with submucosal tumor of the appendix by colonoscopy in that hospital.

Routine laboratory examination on admission did not show any abnormalities, except for tumor markers, e.g. carcinoembryonic antigen, showing a high level. CT scan indicated a $4.0 \mathrm{~cm}$ cystic mass arising from the cecum (fig. 3a). Colonoscopy showed a submucosal 
Yoshida et al:: Two Cases of Mucinous Cystadenoma of the Appendix Successfully

Treated by Laparoscopy

tumor in the cecum. A barium enema study demonstrated displacement on the mesenteric side of the cecum. The suspected preoperative diagnosis was mucinous cystadenoma and the patient received elective laparoscopic surgery. There was no evidence of ascites and peritoneal implants. The operation was performed as in case 1 (fig. 3b). Microscopic examination of the appendix showed multilocular cystadenoma. The postoperative course was uneventful, and the patient had no recurrent disease at 2-year follow-up.

\section{Discussion}

Mucocele of the appendix caused by a retention cyst, mucosal hyperplasia, mucinous cystadenoma or mucinous cystadenocarcinoma is found in $0.2-0.3 \%$ of resected appendices in Europe and the United States $[1,2]$. The external appearance is gross enlargement of an appendix, whose lumen is distended by mucin. Even in benign disease such as cystadenoma, dissemination of mucin-producing cells into the peritoneal cavity can cause pseudomyxoma peritonei. Since its diagnosis in terms of malignancy or benignity is difficult before surgery, it is important to remove it without rupture of the lesion.

Successful laparoscopic management of appendiceal mucocele has been reported. Laparoscopic appendectomy and resection of the appendix with cecum have been demonstrated to confer a faster postoperative recovery course than the open approach [7]. However, it is important to avoid rupture of the mucocele during operation. To achieve this, we fold gauze around the tumor rather than grasping it directly. We achieved successful laparoscopic management by this procedure.

Since we could not rule out the presence of malignancy during the operation, the ileocecal vessels were divided at the root to remove the lymph nodes along the vessels. Lymph node metastasis has been reported in cases of adenocarcinoma of the appendix [5]. As for the extent of tumor resection, recent investigations have revealed that right hemicolectomy provides no survival advantage [8], and appendectomy or cecectomy with a negative margin is preferable [5]. Although pathological diagnosis revealed that the tumor was benign mucinous cystadenoma of the appendix, it was difficult to decide on the extent of lymph node dissection during the operation.

In summary, the present case report describes successful laparoscopic resection of an appendiceal mucocele to a mucinous cystadenoma. This laparoscopic approach avoids a large incision for exploration of the peritoneal cavity and confers the advantage of minimally invasive surgery. It is important to plan careful resection to remove a mass. It was possible to achieve this by using a laparoscopic procedure with gauze technique.

\section{References}

1 Landen S, Bertrand C, Maddern GJ, et al: Appendiceal mucoceles and pseudomyxoma peritonei. Surg Gynecol Obstet 1992;175:401-404.

2 Dhage-Ivatury S, Sugarbaker PH: Update on the surgical approach to mucocele of the appendix. J Am Coll Surg 2006;202:680-684.

3 Behranwala KA, Agarwal T, El-Sharkawi D, et al: Laparoscopic resection of mucinous cystadenoma of appendix: a careful decision. Surg Laparosc Endosc Percutan Tech 2006;16:347-348.

$\checkmark 4$ Lau H, Yuen WK, Loong F, Lee F: Laparoscopic resection of an appendiceal mucocele. Surg Laparosc Endosc Percutan Tech 2001;12:367-370.

5 Matsui H, Igarashi N, Okamura A, et al: Laparoscopy-assisted resection of an appendiceal mucinous cystadenoma. Tokai J Exp Clin Med 2007;32:140-143.

6 González Moreno S, Shmookler BM, Sugarbaker PH: Appendiceal mucocele. Contraindication to laparoscopic appendectomy. Surg Endosc 1998;12:1177-1179. 
Yoshida et al.: Two Cases of Mucinous Cystadenoma of the Appendix Successfully Treated by Laparoscopy

7 Chung RS, Rowland DY, Li P, et al: A meta-analysis of randomized controlled trials of laparoscopic versus conventional appendectomy. Am J Surg 1999;177:250-256.

-8 González-Moreno S, Sugarbaker PH: Right hemicolectomy does not confer a survival advantage in patients with mucinous carcinoma of the appendix and peritoneal seeding. Br J Surg 2004;91:304-311.
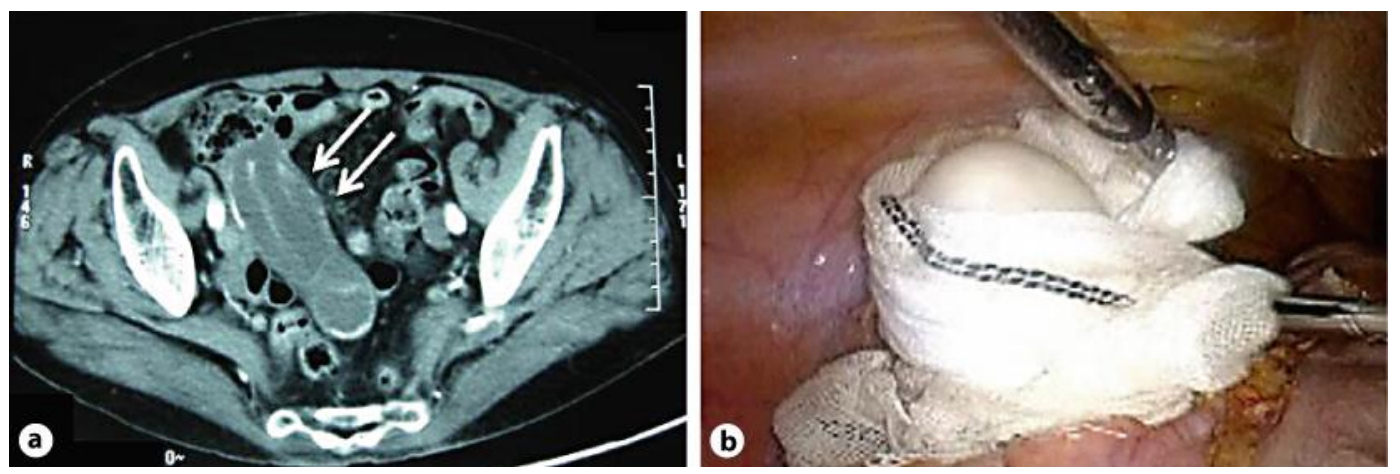

Fig. 1. a CT scan indicated a $10 \times 3.5 \mathrm{~cm}$ cystic mass arising from the cecum with calcification inside (arrows). $\mathbf{b}$ We folded gauze around the tumor rather than grasping it directly.
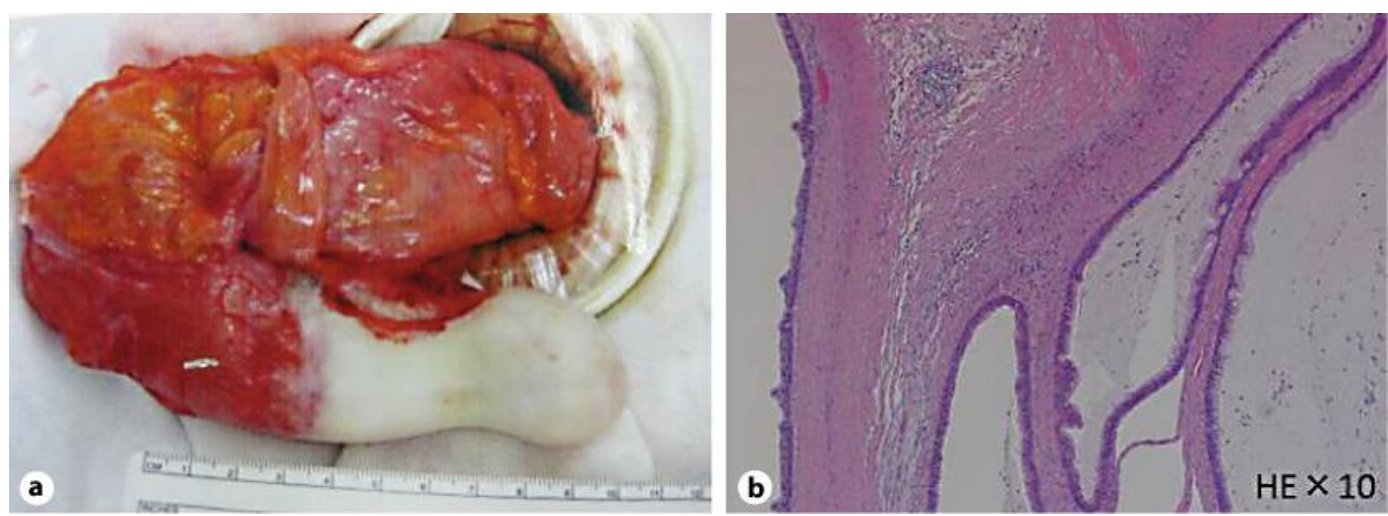

Fig. 2. a The resected specimen demonstrating a $10 \mathrm{~cm}$ mucinous tumor. We used a rap disk to transport the specimen through the abdominal wall. $\mathbf{b}$ Microscopic examination demonstrating atypical epithelial cells filled with mucoid fluid. No malignant cells were identified $(\mathrm{HE}, \times 10)$. 


\begin{tabular}{l|l}
\hline Case Rep Gastroenterol 2013;7:44-48 \\
\hline DOI: $\underline{10.1159 / 000346299}$ & $\begin{array}{l}\odot 2013 \text { S. Karger AG, Basel } \\
\text { www.karger.com/crg }\end{array}$ \\
\hline
\end{tabular}

Yoshida et al.: Two Cases of Mucinous Cystadenoma of the Appendix Successfully Treated by Laparoscopy
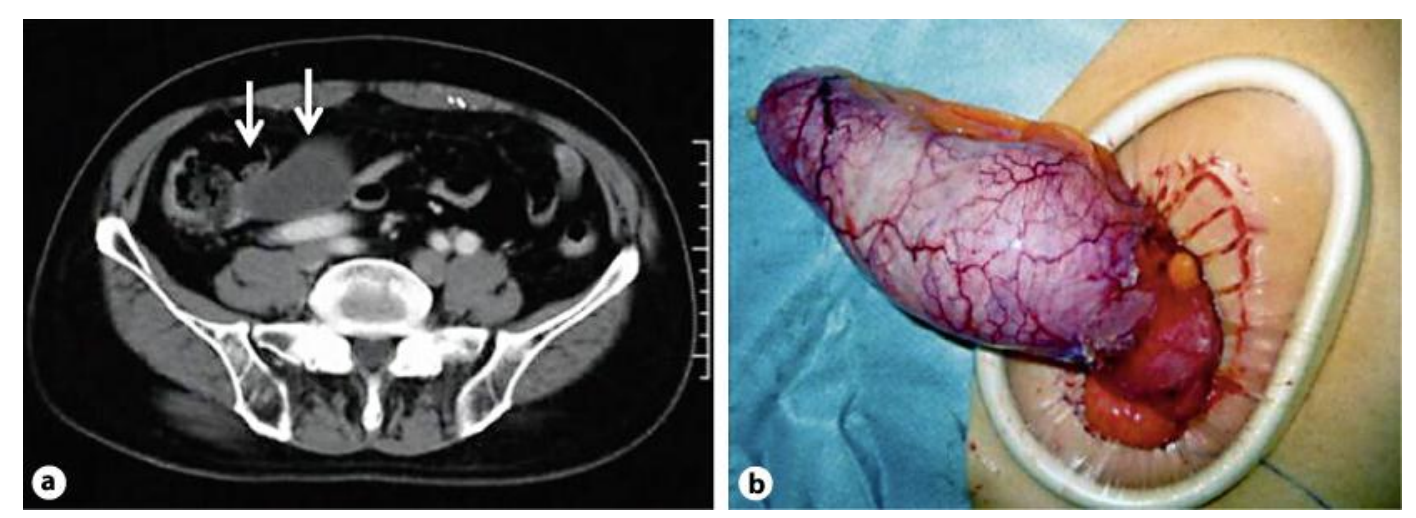

Fig. 3. a CT scan indicated a $4.0 \mathrm{~cm}$ cystic mass arising from the cecum (arrows). b The resected specimen demonstrating an $8 \mathrm{~cm}$ mucinous tumor. We used a rap disk to transport the specimen through the abdominal wall. 